\title{
NEW COMBINED PIXEL/OBJECT-BASED TECHNIQUE FOR EFFICIENT URBAN CLASSSIFICATION USING WORLDVIEW-2 DATA
}

\author{
Ahmed Elsharkawy ${ }^{* a, b}$, Mohamed Elhabiby ${ }^{\mathrm{a}, \mathrm{c}}$ \& Naser El-Sheimy ${ }^{\mathrm{a}, \mathrm{d}}$ \\ ${ }^{a}$ Dept. of Geomatics Engineering, University of Calgary, Calgary, Alberta, T2N 1N4 \\ Phone: 403-210-7897, Fax: 403-284-1980, \\ bEmail: askelsha@ucalgary.ca \\ ${ }^{c}$ Public Works Department, Faculty of Engineering, Ain Shams University, Cairo, Egypt \\ Email: mmelhabi@ucalgary.ca \\ d Email: elsheimy@ucalgary.ca
}

\section{Commission VII/4}

KEY WORDS: High resolution satellite imagery, Pixel/ Object-based, Curvelet transform, Edge detection, Band ratio, Building detection.

\begin{abstract}
The new advances of having eight bands satellite mission similar to WorldView-2, WV-2, give the chance to address and solve some of the traditional problems related to the low spatial and/or spectral resolution; such as the lack of details for certain features or the inability of the conventional classifiers to detect some land-cover types because of missing efficient spectrum information and analysis techniques. High-resolution imagery is particularly well suited to urban applications. High spectral and spatial resolution of WorldView-2 data introduces challenges in detailed mapping of urban features. Classification of Water, Shadows, Red roofs and concrete buildings spectrally exhibit significant confusion either from the high similarity in the spectral response (e.g. water and Shadows) or the similarity in material type (e.g. red roofs and concrete buildings).
\end{abstract}

This research study assesses the enhancement of the classification accuracy and efficiency for a data set of WorldView-2 satellite imagery using the full 8-bands through integrating the output of classification process using three band ratios with another step involves an object-based technique for extracting shadows, water, vegetation, building, Bare soil and asphalt roads. Second generation curvelet transform will be used in the second step, specifically to detect buildings' boundaries, which will aid the new algorithm of band ratios classification through efficient separation of the buildings. The combined technique is tested, and the preliminary results show a great potential of the new bands in the WV-2 imagery in the separation between confusing classes such as water and shadows, and the testing is extended to the separation between bare soils and asphalt roads. The Integrated band ratiocurvelet transform edge detection techniques increased the percentage of building detection by more than $30 \%$.

\section{INTRODUCTION}

The processes of per-pixel supervised classification methods were always the primary tool to extract land cover classes from digital remotely sensed data (Bhaskaran et al., 2010). The ultimate goal of any image classification procedure is to automatically categorize all pixels in an image into land cover classes (Lillesand and kiefer, 2001). For the purpose of urban planning, supervised classification has been used extensively. Unfortunately, this procedure always results in mixed pixel's problem (Bhaskaran et al., 2010). This problem leads many researchers to incorporate segmentation, texture, context, colour, and many other parameters to glide the mixed or wrongly classified pixels into their proper classes. Segmentation can be done either by detecting similarities or by detecting singularities (edge detection) (Gonzalez and Woods, 2002). Contrasting spectral methods, object-oriented methods are based on segmenting the image into homogeneous parcels of pixels then these parcels are classified using spectral, spatial, textural, relational and contextual methods (Bhaskaran et al., 2010).

The primary objective of this study was to classify urban features from a WorldView-2 imagery by using both per-pixel classification (three new band ratios), and object-oriented classification method (edge detection using curvelet transforms). The first approach was to use the three new band ratios to classify the image and check accuracy of all classes. The following approach was to improve the accuracy of the lowest two classes' accuracy.

In 2009 Digital Globe launched the WorldView-2 satellite. It is the first commercial high-resolution satellite to provide eight spectral sensors in the visible to the near-infrared range. Each sensor closely focuses on a particular range of the electromagnetic spectrum which is sensitive to a specific feature on the ground and was designed to improve the segmentation and classification of land and marine. Figure 1 is a concise comparison between QuickBird, IKONOS, WorldView-1 and WorldView-2 of their spectral and panchromatic bands.

In addition to its large-scale collection capacity, WorldView-2 has a high spatial and spectral resolution. According to (Globe, 2009) this satellite can capture a $46 \mathrm{~cm}$ panchromatic image with $1.84 \mathrm{~m}$ spectral resolution with 8-band multispectral imagery. The high spatial resolution facilitates the differentiation of fine details like small and medium-size buildings, shallow reefs and individual trees while the high spectral resolution provides detailed information about diverse 
areas such as road surface quality, ocean depth, and plant health(Globe, 2009). Taking advantage of all these properties a new pixel/object-based technique is introduced to extract shadows, water, vegetation, buildings, bare soil and asphalt roads for a complex scene in Ismailia city, $120 \mathrm{Kms}$ to the north-east of Cairo the capital of Egypt. The new technique integrates the classification result from three new band ratios along with edge detection algorithm using the second generation curvelet transform(Elsharkawy et al., 2011b). The band ratio section showed very good results regarding water, shadow, asphalt roads, vegetation. While this approach gives relatively poor results distinguishing between buildings and bare soil due to the great similarity in spectral responses of these two classes. Curvelet transforms will be implemented as an edge detector to extract building parcels. The output of the last step will be integrated with the classification results to enhance the overall accuracy.

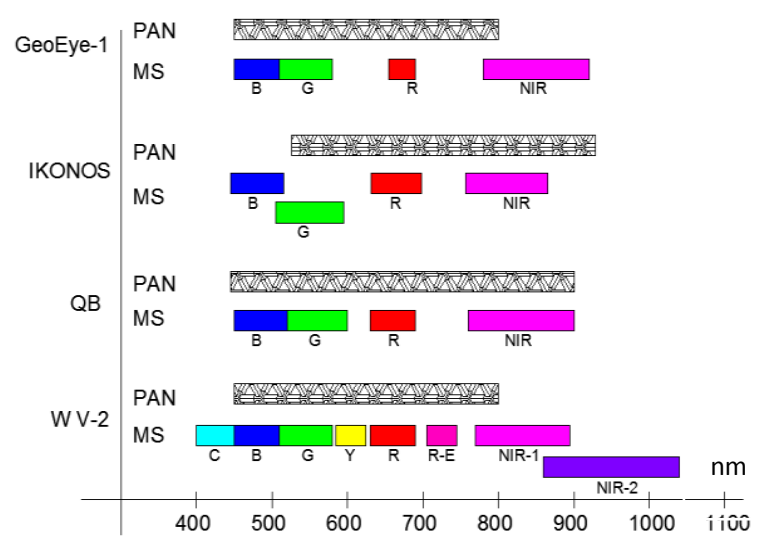

Figure 1 Panchromatic and multispectral wavelengths for different satellites, (Elsharkawy et al., 2011a)

Generally, The new spectral bands in WorldView-2, Coastal blue, Yellow, Red edge and NIR-2, are targeting costal and vegetation land cover types with applications in plant species identification, mapping of vegetation stress and crop types, wetlands, coast water quality, and bathymetry (Marchisio et al., 2010) . To be more specific, the Yellow and Red edge bands are filling important gaps in the spectrum that relate to the ability of capturing vegetation (Shafri et al., 2006). Moreover, Coastal blue and NIR2 bands are very helpful for discriminating among different types of vegetation and many man-made objects (Herold et al., 2002).

\section{DATA AND METHOD}

\subsection{Area of study}

The study area is a residential area in Ismailia city about 120 $\mathrm{Km}$ to the northeast direction from Cairo the capital of EGYPT. The study area is an urban area comprises scattered buildings, roads, vegetation areas, shadowed areas, shoreline and water body. The data was provided by Digital Globe, http://www.digitalglobe.com; the images were captured on April 7th, 2011 in morning time. Figure 2 illustrates false-color composite, NIR-1 G B of part of the study area.

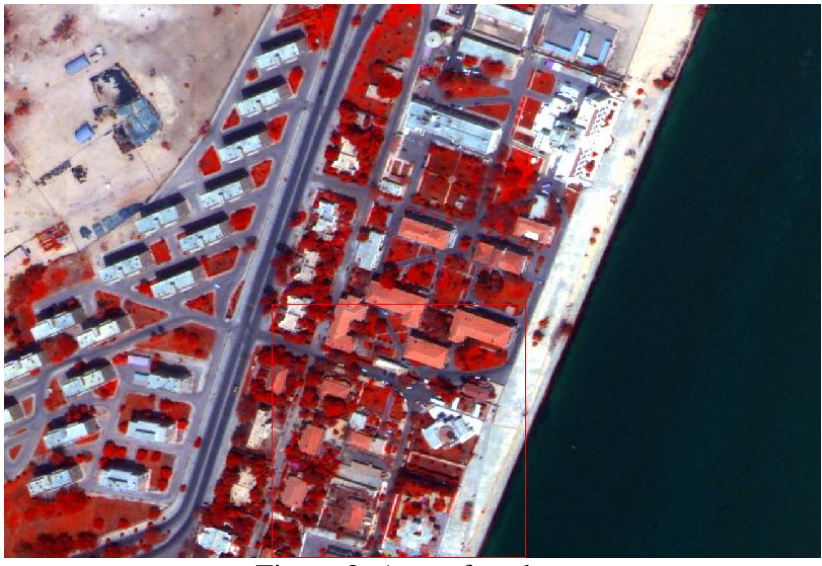

Figure 2. Area of study

\subsection{Methodology}

The proposed algorithm begins with a data fusion between the panchromatic band of the WorldView data, $0.50 \mathrm{~m}$, and the multispectral ones, $2.00 \mathrm{~m}$ resolution, to generate 8 -spectral bands with a resolution of $0.50 \mathrm{~m}$. One of the most common fusion techniques is the Brovey Transform. This technique is optimum when contrast in shadows, water, and high reflectance areas such as urban features is needed. The procedure of this transform starts with multiplying each multi spectral band by the high-resolution panchromatic band, and then divides each product by the sum of the multi spectral bands. Since the Brovey Transform is intended to produce RGB images, only three bands at a time should be merged from the input multispectral scene (Nikolakopoulos, 2008) in our case, we choose NIR-1, R and Y bands. The next step is applying a Gaussian high-pass filter to enhance the edges. Based on the curvelet transform theory an implementation for detecting edges will be introduced depending on the fact that the values of curvelet coefficients are determined by how they are aligned in the real image, the more accurately a curvelet is aligned with a given curve in an image; the higher is its coefficient value.

Each scale level will contain different information related to the size and shape of the edges. Consequently, by arranging the coefficients of each level from the higher to the lower values and take the most significant part of them, this will enhance the edge information that represents the important part of the image to us. Then, the coefficients are reconstructed to get a new image where the edge parts are enhanced.

Morphological filters will be applied to remove the undesired noised pixels. After that, a filling process will be used to generate the candidate parcels, as in figure 7 , where buildings will be extracted from it in a final step.

The final step involves calculation of statistics for the enclosed boundaries; such as area, major and minor axis and solidity. Based on shape and area characteristics, buildings will be extracted from the candidate parcels. The algorithm is summarized in figure 3; more details about this technique can be found in (Elsharkawy et al., 2011b) . 


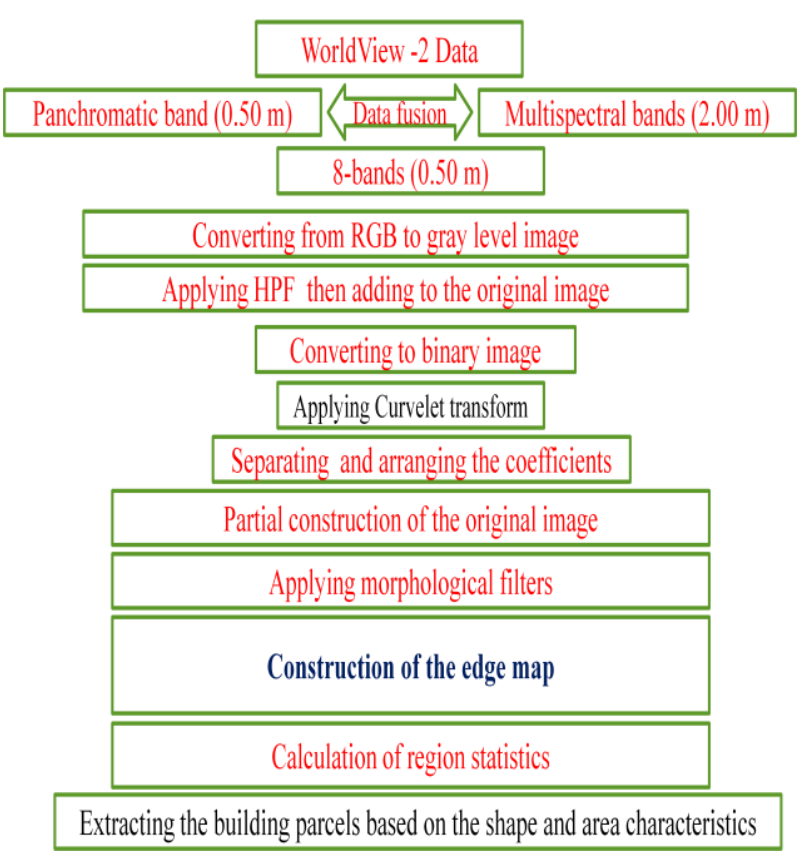

Figure 3. The algorithm for buildings extraction using curvelet transforms

The second step, of the algorithm deals with the classification step. In this step a multi-layer classification process based on new band ratios will be applied to extract seven classes (water, vegetation, bare soil, Asphalt, Shadows, Buildings)(Elsharkawy et al., 2012)

The traditional NDVI ratio plus two new band ratios are introduced, first one the NDVI (R1) especially suited for vegetation and water; second ratio (R2) was used to detect asphalt, man-made object and shadow. Finally (R3) was applied to detect bare soil and buildings. Table 1 summarizes the three ratios and their usage.

\begin{tabular}{|c|c|c|}
\hline & Band ratio & Target classes \\
\hline \multirow{2}{*}{$\mathbf{R 1}$} & $R-N I R 1$ & Vegetation \\
\hline & $\overline{R+N I R 1}$ & Water \\
\hline \multirow{3}{*}{$\mathbf{R 2}$} & $C-R$ & Asphalt \\
\hline & $\frac{\pi}{C+R}$ & Shadow \\
\hline & $C+R$ & Manmade object \\
\hline \multirow{2}{*}{$\mathbf{R 3}$} & $N I R 1-Y$ & Building \\
\hline & $N I R 1+Y$ & Barren \\
\hline
\end{tabular}

Table 1. Band ratios and their implementation

$\mathrm{R} 1$ is applied first to separate the image into vegetation and non vegetation, and then $\mathrm{R} 2$ is applied to detect the water above a certain threshold. Asphalt, shadow and red roofs are detected within lower and upper thresholds; finally, the R3 ratio is applied to detect bright surfaces below certain threshold. Figure 4 summarizes theses steps.

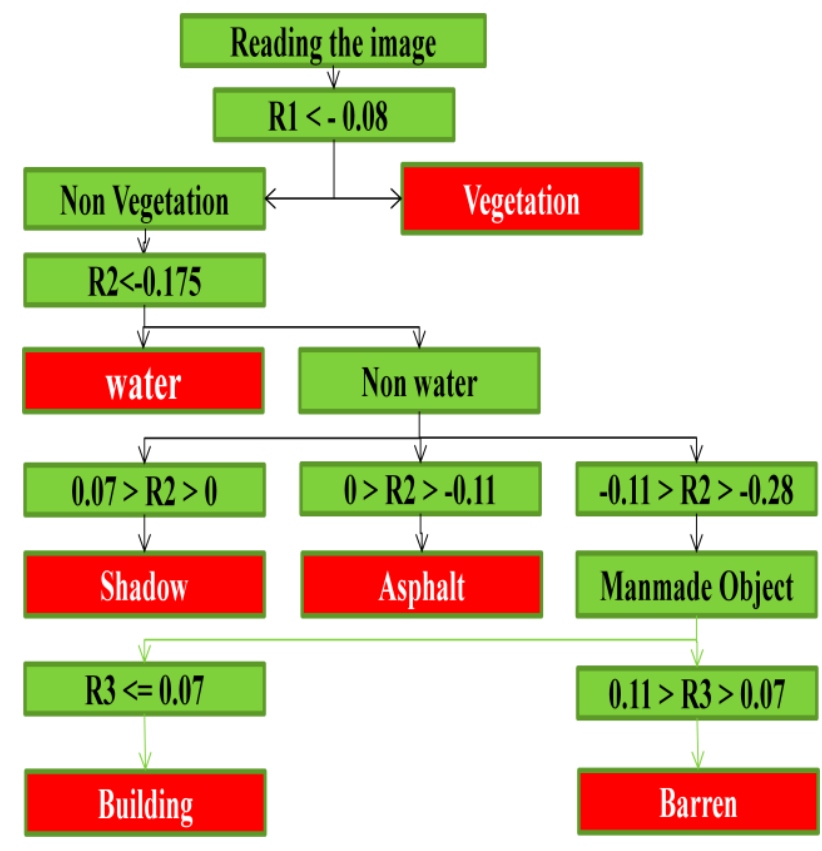

Figure 4. Applying the band ratios with the proposed thresholds

The final step involves the integration between the previous two steps to enhance the pixel-based classification results. The main idea behind this step is to incorporate the object-based results as a classification layer to be added to the multi-layer classification process. In this integration step, we have confidence in the water, vegetation, asphalt and shadow classes, while building and bare soil classes can be modified according to the edge detection process. If we denote the pixel-based classification results by $\mathrm{p}(\mathrm{m}, \mathrm{n})$ and object-based classification results as $E(m, n)$ and the final classification results as $f(m, n)$ so we can apply the following algorithm to integrate the object and pixel based results.

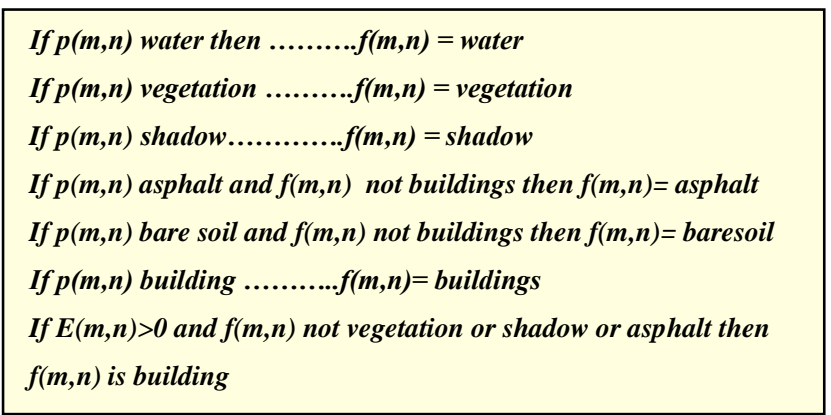

Generally, any imagery will be used in a radiometric/spectral analysis must be converted to spectral radiance at a minimum or top of atmosphere reflectance in order to account for the variation in the relative positions between the sun, the earth and the satellite to obtain absolute values for the NDVI ratios can be applied in any other scene (Updike and Comp, 2010). Converting the Digital Numbers (DN) to Top of Atmosphere (ToA) reflectance is a two-step process. First DNs are converted to ToA radiance values. Then these radiance values are then converted to reflectance values(Elsharkawy et al., 2012; Observation, 2010). 


\section{RESULTS AND ANALYSIS}

Figure 5 illustrates the output from the multi-layer classification process. While figure 6 and 7 represents the edge detection map for the study area and the candidate parcels as buildings respectively.

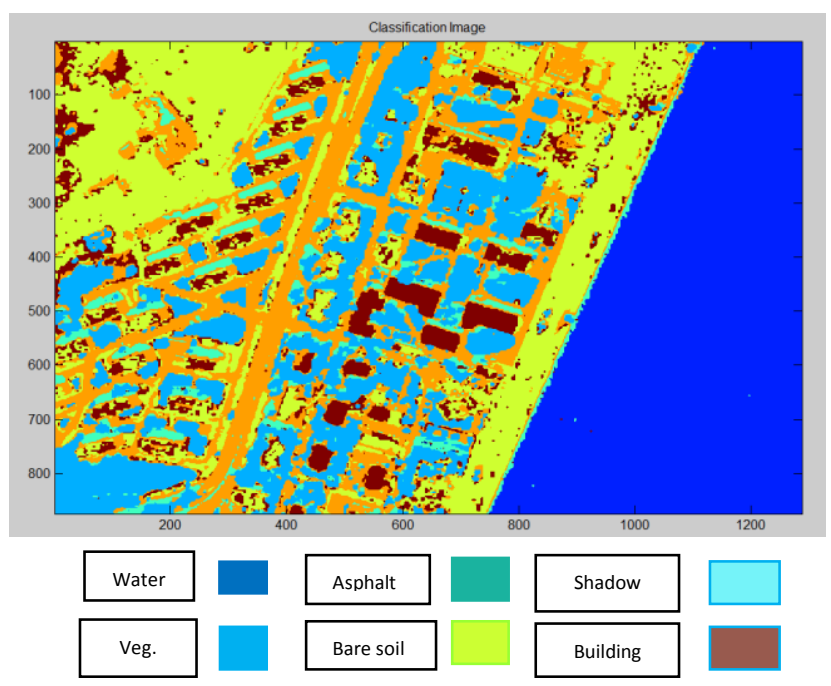

Figure 5. Classification results for the proposed band ratios

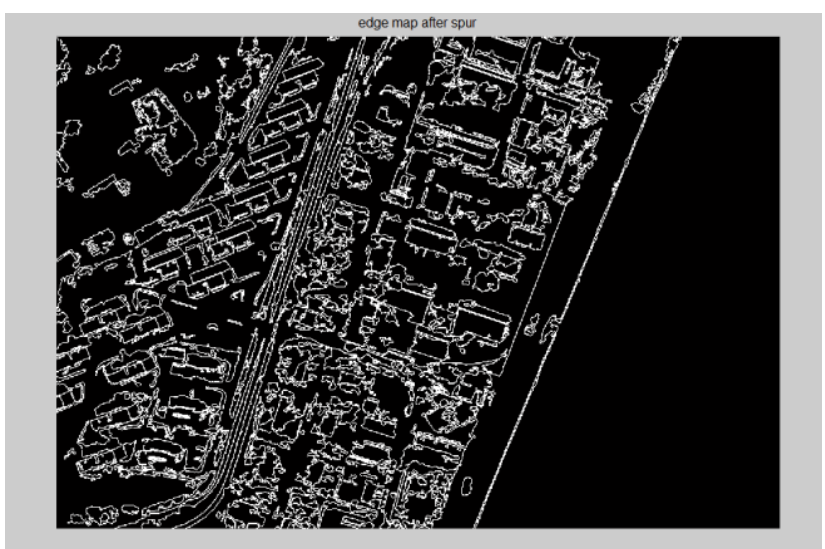

Figure 6. Edge detection result

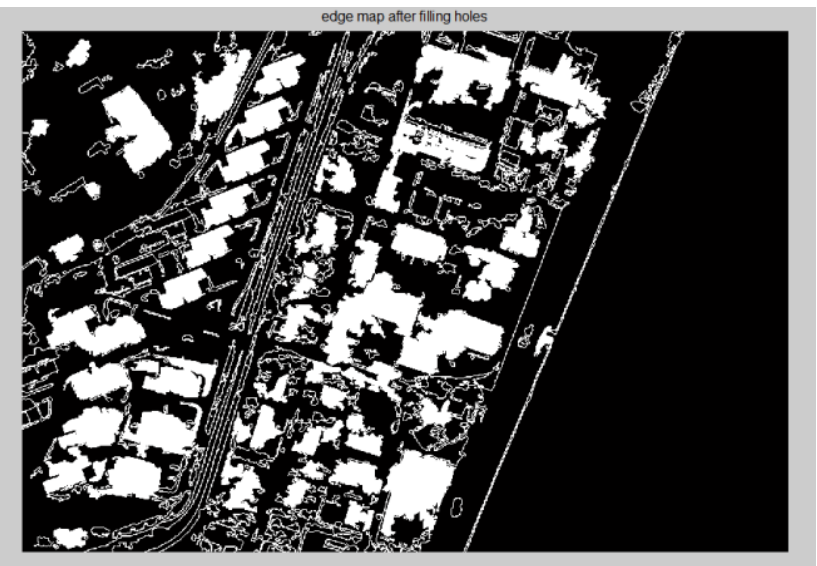

Figure 7. Candidate parcels as buildings
It is quite clear from figure 7 that the algorithm mixed between shadow and vegetation areas with buildings. The integration part will account for this confusion as vegetation and shadow areas are well defined in the multi-layer classification part. A quantitative analysis for the building class (extracted) were done by comparing the number of buildings in the original image by the complete detected number of buildings being detected in the classified images either from pixel-based output or from the final integrated output. Table 2 summarizes the comparison results.

\begin{tabular}{|ccccc|}
\hline & $\begin{array}{c}\text { No. of Red } \\
\text { Roofs }\end{array}$ & $\begin{array}{c}\text { No. of Tall } \\
\text { buildings }\end{array}$ & $\begin{array}{c}\text { No. of Short } \\
\text { buildings }\end{array}$ & Total no. \\
\hline $\begin{array}{c}\text { Ground } \\
\text { truth }\end{array}$ & 14 & 17 & 17 & 48 \\
\hline $\begin{array}{c}\text { Pixel-based } \\
\text { result }\end{array}$ & 12 & 9 & 1 & 22 \\
\hline $\begin{array}{c}\text { Accuracy } \\
\text { \% }\end{array}$ & $\mathbf{8 5 . 7}$ & $\mathbf{5 2 . 9}$ & $\mathbf{5 . 8}$ & $\mathbf{4 5 . 8}$ \\
\hline $\begin{array}{c}\text { Proposed } \\
\text { method }\end{array}$ & 14 & 16 & 11 & 41 \\
\hline $\begin{array}{c}\text { Accuracy } \\
\text { \% }\end{array}$ & $\mathbf{1 0 0}$ & $\mathbf{9 4 . 1}$ & $\mathbf{6 4 . 7}$ & $\mathbf{8 5 . 4}$ \\
\hline
\end{tabular}

Table 2. Comparison of results

The final result after integrating the two approaches is in figure 8.

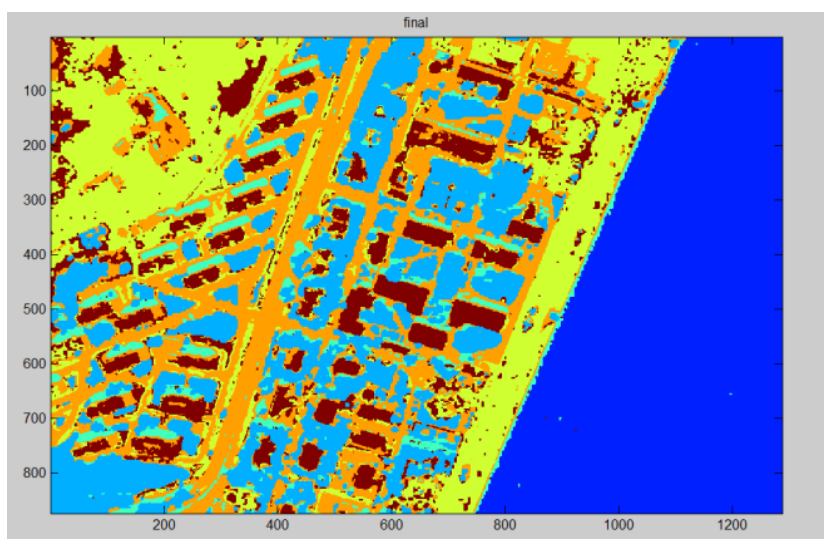

Figure 8 final classification results

\section{CONCLUSION}

A method using both pixel and object-based classification process was introduced to extract six land covers; vegetation, water, buildings, shadows, asphalt roads and bare soil. WorldView-2 imagery over Ismailia city, Egypt was used. The classification accuracy for five classes out of six was acceptable, and it was enhanced for buildings' class. Specifically, buildings' class accuracy was enhanced from $45.8 \%$ to $85.4 \%$ using this technique. This technique was applied for many other parts of the large scene, Ismailia city, and the results showed great potentials of using this method in enhancing the percentage of the detected buildings.

\section{ACKNOWLEDGMENTS}

This work was supported in part by research funds from TECTERRA Commercialization and Research Centre, the 
Canada Research Chairs Program, and the Natural Science and Engineering Research Council of Canada (NSERC).

Many thanks for the Digital Globe for providing the necessary data for this research paper.

\section{REFERENCES}

Bhaskaran, S., S. Paramananda, and M. Ramnarayan. 2010. Per-Pixel and Object-Oriented Classification Methods for Mapping Urban Features Using Ikonos Satellite Data. Applied Geography. 30(4):650-665

Elsharkawy, A., M. Elhabiby, and N. El-Sheimy, 2011a. A Modified Parallelepiped-Like Method for Supervised Classification for High Resolution Satellite Imagery $C G U$ Annual Scientific Meeting. Banff, Calgary. pp. 4.

Elsharkawy, A., M. Elhabiby, and N. El-Sheimy. 2011b. Second Generation Curvelet Transform for Building Extraction from High Resolution Satellite Imagery. GEOMATICA. 65(4):387-399

Elsharkawy, A., M. Elhabiby, and N. El-Sheimy. 2012. Improvement in the Detection of Land Cover Classes Using the Worldview-2 Imagery ASPRSSacremento, CA, pp. 11.

Globe ,D. (2009) "The Benefits of the 8 Spectral Bands of Worldview-2," In: White Paper, Digital Globe, 12

Gonzalez, R. C., and R. E. Woods (2002) Digital Image Processing, Prentice Hall

Herold, M., M. Gardner, B. Hadley, and D. Roberts. 2002. The Spectral Dimension in Urban Land Cover Mapping from Highresolution Optical Remote Sensing Data. The 3rd Symposium on Remote Sensing of Urban Areas. 8

Lillesand, T. M., and R. W. kiefer (2001) Remote Sensing and Image Interpretation, 4th Ed., John Wiley and Sons, Inc.

Marchisio, G., F. Pacifici, and C. Padwick. 2010. On the Relative Predictive Value of the New Spectral Bands in the Worldwiew-2 Sensor. Geoscience and Remote Sensing Symposium (IGARSS), 2010 IEEE International25-30 July 2010. pp. 2723-2726.

Nikolakopoulos, K. G. 2008. Comparison of Nine Fusion Techniques for Very High Resolution Data. Photogramm. Eng. Remote Sens. 74(5):647-659

Observation, T. Y. C. f. E., 2010. Converting Digital Numbers to Top of Atmosphere (Toa) Reflectance,

http://www.yale.edu/ceo,
Shafri, H., M. Salleh, and A. Ghiyamat. 2006. Hyperspectral Remote Sensing of Vegetation Using Red Edge Position Techniques. American Journal of Applied Sciences. 3(6)(18641871

Updike, T., and C. Comp, 2010. Radiometric Use of Worldview-2 Imagery. Nov. 1st , 2010. 